University of Windsor

Scholarship at UWindsor

Physics Publications

Department of Physics

1981

\title{
Application of discrete-basis-set methods to the Dirac equation
}

Gordon W. F. Drake

University of Windsor

S. P. Goldman

Follow this and additional works at: https://scholar.uwindsor.ca/physicspub

Part of the Physics Commons

\section{Recommended Citation}

Drake, Gordon W. F. and Goldman, S. P.. (1981). Application of discrete-basis-set methods to the Dirac equation. Physical Review A, 23 (5), 2093-2098.

https://scholar.uwindsor.ca/physicspub/97

This Article is brought to you for free and open access by the Department of Physics at Scholarship at UWindsor. It has been accepted for inclusion in Physics Publications by an authorized administrator of Scholarship at UWindsor. For more information, please contact scholarship@uwindsor.ca. 


\title{
Application of discrete-basis-set methods to the Dirac equation
}

\author{
G. W. F. Drake and S. P. Goldman \\ Department of Physics, University of Windsor, Windsor, Ontario, Canada N9B $3 P 4$
}

(Received 21 November 1980)

\begin{abstract}
Variational solutions to the Dirac equation in a discrete $L^{2}$ basis set are investigated. Numerical calculations indicate that for a Coulomb potential, the basis set can be chosen in such a way that the variational eigenvalues satisfy a generalized Hylleraas-Undheim theorem. A number of relativistic sum rules are calculated to demonstrate that the variational solutions form a discrete representation of the complete Dirac spectrum including both positiveand negative-energy states. The results suggest that widely used methods for constructing $L^{2}$ representations of the nonrelativistic electron Green's function can be extended to the Dirac equation. As an example, the relativistic basis sets are used to calculate electric dipole oscillator strength sums from the ground state, and dipole polarizabilities.
\end{abstract}

\section{INTRODUCTION}

In nonrelativistic quantum mechanics, variational methods provide a powerful and widely used technique for the construction of approximate eigenvalues and eigenfunctions of the Schrödinger equation. To briefly review, if $\psi_{\mathrm{tr}}$ is any normalizable trial function and $H$ is the Schrödinger Hamiltonian, then the expression

$$
E_{\mathrm{tr}}=\left\langle\psi_{\mathrm{tr}}|H| \psi_{\mathrm{tr}}\right\rangle /\left\langle\psi_{\mathrm{tr}} \mid \psi_{\mathrm{tr}}\right\rangle
$$

is an upper bound to the ground-state energy. If $\psi_{\mathrm{tr}}$ is expanded in an orthonormal basis set $\Phi_{i}$ with linear variational coefficients $a_{i}$,

$$
\psi_{\mathrm{tr}}=\sum_{i=1}^{N} a_{i} \Phi_{i} .
$$

Then $E_{\mathrm{tr}}$ is optimized with respect to the $a_{i}$ by solving the set of $N$ homogeneous equations

$$
\frac{\partial E_{\mathrm{tr}}}{\partial a_{i}}=0
$$

for the $a_{i}$. This is equivalent to diagonalizing the $N \times N$ matrix $H$ with matrix elements

$$
H_{i j}=\left\langle\Phi_{i}|H| \Phi_{j}\right\rangle \text {. }
$$

The $j$ th eigenvector $\left|\Psi_{j}\right\rangle$ gives the optimum values of the $a_{i}^{(j)}$ and, by the Hylleraas-Undheim theorem, ${ }^{1}$ the $N$ eigenvalues are upper bounds to the true eigenvalues of $H$. The bounds progressively improve as $N$ is increased since the matrix eigenvalues necessarily move downward (or remain fixed).

The bounds discussed above cannot in general be extended to the Dirac Hamiltonian $H_{D}$ because, unlike $H, H_{D}$ is not bounded from below. Any positive-energy eigenvalue of the matrix $H_{D}$ corresponding to (1.4) can collapse without limit to a negative- energy eigenvalue as the basis set is enlarged.

Variational methods have previously been used to solve the Dirac-Hartree-Fock equations ${ }^{2,3}$ and the stability of these solutions against collapse to negative-energy states has recently been discussed. ${ }^{4}$ In this context, stability is ensured by projecting out the negative-energy states. In the present work, we obtain instead a variational representation of the complete Dirac spectrum without the explicit use of projection operators. We suggest on the basis of numerical evidence that for the special case of the Dirac equation with a Coulomb potential, the basis set can be chosen in such a way that the eigenvalues do yield upper bounds to the discrete positive energies, while the negative eigenvalues lie in the negativeenergy continuum.

The primary usefulness of the results is that the $N$-eigenvalue spectrum of $H_{D}$ forms a discrete variational representation of the actual eigenvalue spectrum of $H_{D}$, including both positive and negative energies. This allows the Dirac Green's operator to be approximated by the expression

$$
\left(H_{D}-z\right)^{-1} \simeq \sum_{j=1}^{N} \frac{\left|\psi_{j}\right\rangle \psi_{j} \mid}{E_{j}-z},
$$

where the sum over $j$ includes both positive and negative variational eigenvalues of $H_{D}$. The nonrelativistic analog of (1.5) has been widely used to perform summations over complete sets of intermediate states, ${ }^{5}$ and to extract information on scattering states. ${ }^{6,7}$ The present results suggest that the same techniques can be extended to the Dirac Hamiltonian.

In the remainder of the paper, the variational solution of the Dirac equation is first discussed in Sec. II. We then show the variational eigenvalues are bounds on the exact eigenvalues in Sec. III, and suggest on the basis of numerical evidence that a generalized Hylleraas-Undheim theorem exists for the relativistic Coulomb problem. The completeness of the basis set is tested by calculating a number of sum rules in Sec. IV. The variational basis set is then used in Sec. V 
to calculate the electric dipole oscillator-strength sum from the ground state.

\section{VARIATIONAL FORMULATION}

We restrict our discussion to an electron in a Coulomb potential $v(r)=-Z e^{2} r$ since this has special properties which lead to bounds. The four-component Dirac equation is then

$$
H \psi=E \psi
$$

with

$$
H=c \vec{\alpha} \cdot \overrightarrow{\mathrm{p}}+\beta m c^{2}-\frac{Z e^{2}}{r}
$$

and the Dirac matrices $\overline{\vec{\alpha}}$ and $\beta$ have their usual meanings. For any central potential, the solutions to $(2.1)$ can be written in the form ${ }^{8}$

$$
\psi=\left(\begin{array}{cc}
i g r^{-1} & \Omega_{j l M} \\
-f r^{-1} & \Omega_{j i} M
\end{array}\right), \quad \tilde{l}=2 j-l
$$

where $g(\boldsymbol{r})$ and $f(\boldsymbol{r})$ are the large and small radial functions and $\Omega_{j l M}$ is a two-component spherical spinor defined as the vector coupled product

$$
\Omega_{j l M}=\sum_{m, \mu}\left\langle l m \frac{1}{2} \mu \mid j M\right\rangle Y_{l}^{m}(\theta, \varphi) \chi_{\mu}
$$

with

$$
\chi_{1 / 2}=\left(\begin{array}{l}
1 \\
0
\end{array}\right) \text { and } \chi_{-1 / 2}=\left(\begin{array}{l}
0 \\
1
\end{array}\right)
$$

For convenience, we define a real two-component radial spinor by

$$
\Phi(r)=\left(\begin{array}{l}
g(r) \\
f(r)
\end{array}\right) .
$$

Then $\Phi(r)$ satisfies the radial Dirac equation

$$
H_{\boldsymbol{r}} \Phi=\alpha E \Phi
$$

with

$$
H_{r}=-i \sigma_{y} \frac{d}{d r}+\sigma_{x} \frac{\kappa}{r}+\frac{\sigma_{\varepsilon}}{\alpha}-\frac{\alpha Z}{r}
$$

in atomic units $(e=\hbar=m=1)$. The $\sigma$ 's are the Pauli spin matrices, and $\kappa$ is the Dirac quantum number $\kappa= \pm\left(j+\frac{1}{2}\right)$ for $j=l \mp \frac{1}{2}$ 。 The exact positive-energy solutions to (2.6) for quantum numbers $n$ and $\kappa$ can be written in the form

$$
\Phi_{n, k}=r^{\gamma-1} e^{-\lambda r} \sum_{i=1}^{N} r^{i}\left[a_{i}\left(\begin{array}{l}
1 \\
0
\end{array}\right)+b_{i}\left(\begin{array}{l}
0 \\
1
\end{array}\right)\right]
$$

with

$$
\gamma=\left(\kappa^{2}-\alpha^{2} Z^{2}\right)^{1 / 2}
$$

and

$$
\lambda=\frac{Z}{\left[n^{2}-2(n-|\kappa|)(|\kappa|-\gamma)^{1 / 2}\right]^{1 / 2}} .
$$

Our variational procedure consists of using a trial function of the same form as $(2.8)$ with $a_{i}$ and $b_{i}$ regarded as linear variational parameters, and $\lambda$ a nonlinear parameter which can be adjusted arbitrarily. Starting with $2 N$ basis functions

$$
\begin{aligned}
& g_{i}=r^{\gamma+i-1} e^{-\lambda r}\left(\begin{array}{l}
1 \\
0
\end{array}\right), \\
& f_{i}=r^{\gamma+i-1} e^{-\lambda r}\left(\begin{array}{l}
0 \\
1
\end{array}\right), \quad i=1,2, \ldots, N
\end{aligned}
$$

the computational procedure is first to orthonormalize the basis set, and then to diagonalize $H_{r}$ to obtain the linear combinations

$$
\Phi_{i}=\sum_{j=1}^{N}\left(a_{i, j} g_{j}+b_{i, j} f_{j}\right)
$$

which satisfy

$$
\begin{aligned}
& \int_{0}^{\infty} \Phi_{i}^{\dagger} \Phi_{j} d r=\delta_{i, j}, \\
& \int_{0}^{\infty} \Phi_{i}^{\dagger} H_{r} \Phi_{j} d r=\alpha \epsilon_{i} \delta_{i, j} .
\end{aligned}
$$

All necessary integrals can easily be evaluated analytically. The $\epsilon_{i}, i=1, \ldots, 2 N$ are the discrete variational eigenvalues.

\section{BOUNDS}

In general, the $\epsilon_{i}$ obtained above are distributed between $+\infty$ and $-\infty$, and move in an unpredictable way as the parameters of the basis set are varied. There is no guarantee that any eigenvector is an approximate representation of some particular state, and the eigenvalues are not bounds. But for the special case of a Coulomb potential, it appears that one does obtain bounds in the form of a generalized Hylleraas-Undheim Theorem. We give here a rigorous proof for the case $N=1$, together with numerical evidence that the theorem can be extended to arbitrary $N$.

For the case $N=1$, consider a trial function of the form

$$
\Phi_{\mathrm{tr}}=g(r)\left[a\left(\begin{array}{l}
1 \\
0
\end{array}\right)+b\left(\begin{array}{l}
0 \\
1
\end{array}\right)\right],
$$

where $g(r)$ is an arbitrary continuous function subject to the conditions

$$
\begin{aligned}
& \int_{0}^{\infty} g(r)^{2} d r=1, \\
& \lim _{r \rightarrow 0} g(r)=0 .
\end{aligned}
$$


Then variation of

$$
\alpha E_{\mathrm{tr}}=\int_{0}^{\infty} \Phi_{\mathrm{tr}}^{\dagger} H_{r} \Phi_{\mathrm{tr}} d r / \int_{0}^{\infty} \Phi_{\mathrm{tr}}^{\dagger} \Phi_{\mathrm{tr}} d r
$$

with respect to $a$ and $b$ for an arbitrary potential $v(r)$ yields

$$
E_{\mathrm{tr}}=V \pm \frac{1}{\alpha^{2}}\left(1+\alpha^{2} \kappa^{2} W^{2}\right)^{1 / 2}
$$

where

$$
V=\int_{0}^{\infty} g(r)^{2} v(r) d r
$$

and

$$
W=\int_{0}^{\infty} g(r)^{2} r^{-1} d r .
$$

Clearly, a $g(r)$ can be chosen to yield a wide range of eigenvalues if $V$ and $W$ are independent. But for the Coulomb potential, $V=-Z W$ and (3.4) reduces to

$$
E_{\mathrm{tr}}=-Z W \pm \frac{1}{\alpha^{2}}\left(1+\alpha^{2} \kappa^{2} W^{2}\right)^{1 / 2} .
$$

The lower root always lies below $-1 / \alpha^{2}$, and is therefore a lower bound on the highest negativeenergy eigenvalue. The upper root can be optimized with respect to arbitrary variations in $W>0$ to yield a single minimum

$$
E_{\mathrm{tr}}=\frac{\gamma}{\alpha^{2}|\kappa|}
$$

at

$$
W=Z / \gamma|\kappa| \text {. }
$$

These are the exact values for the lowest positive-energy state having any $\kappa<0$ (i.e., $j=l+\frac{1}{2}$ ). For $\kappa>0$, the above procedure yields a spurious root degenerate with the corresponding state with $\kappa<0$. For example, a $1 p_{1 / 2}(\kappa=1)$ root is obtained which is degenerate with $1 s_{1 / 2}(\kappa=-1)$. This causes no problem as the basis set is enlarged because the single spurious root can be simply discarded. The essential point is that a lowest positive-energy root always exists which prevents the spectrum from collapsing. For $\kappa<0$, the root is a rigorous upper bound on the lowest positive eigenvalue for any choice of $g(r)$ subject to conditions (3.2).

We have not yet been able to extend the formal proof of bounds to basis sets with $N>1$. However, we have done extensive numerical calculations with progressively larger basis sets of the form (2.8) for $\kappa= \pm 1, \pm 2, N$ up to 16 , and a range of values of $\lambda$. In every case, the $2 N$ eigenvalues split into $N$-positive eigenvalues and $N$-negative eigenvalues. The negative eigenvalues all lie in the negative -energy continuum below $E=-1 / \alpha^{2}$, while the positive-energy eigenvalues behave exactly as if the Dirac Hamiltonian were a positive-definite operator. They move progressively lower as $N$ is increased, but never cross the exact energies. The $s_{1 / 2}$ eigenvalues for $Z=92$ and $\lambda=65.2$ are shown for progressively larger basis sets in Fig. 1. The spurious root for $\kappa>0$ discussed previously is always present, but causes no difficulties.

The above behavior depends on there being as many $f_{i}\left[\right.$ (Eq. 2.12)] as $g_{i}[$ (Eq. 2.11)] functions in the basis set. As $f_{i}$ functions are omitted, negative eigenvalues progressively disappear and positive eigenvalues fall progressively below the exact values. It, therefore, appears that for the Coulomb-Dirac Hamiltonian, there exists a generalized Hylleraas-Undheim theorem stating that for $2 \mathrm{~N}$-dimensional basis sets containing as many functionally equivalent degrees of freedom in the upper component as in the lower component, there are $N$-positive eigenvalues and $N$-negative eigenvalues. For $\kappa<0$, the positive eigenvalues are upper bounds on the first $N$ discrete energies. For $\kappa>0$, one obtains bounds on the first $N-1$ discrete energies, together with a spurious root. In both cases, the negative eigenvalues are lower bounds on the highest negative-energy state (i.e., $\left.-m c^{2}\right)$.

\section{THE DIRAC GREEN'S OPERATOR AND SUM RULES}

To the extent that the discrete variational spectrum obtained by diagonalizing $H_{D}$ in a finite basis set represents the actual spectrum of $H_{D}$, the Green's operator can be approximated by

$$
G(z) \simeq \sum_{n=1}^{2 N} \frac{\left|\psi_{n}\right\rangle\left\langle\psi_{n}\right|}{\epsilon_{n}-z},
$$

where the $\epsilon_{n}$ are the variational eigenvalues and the $\left|\psi_{n}\right\rangle$ the corresponding eigenvectors. To test the validity of (4.1), we have evaluated a number of sum rules of the form

$$
S_{i}=\sum_{n}\left(E_{n}-E_{0}\right)^{i}\left|\left\langle\psi_{0}|\overrightarrow{\mathbf{r}}| \psi_{n}\right\rangle\right|^{2},
$$

where the sum includes integrations over both positive- and negative-energy continua. By replacing factors of $\left(E_{n}-E_{0}\right)$ with $\left[H_{D}, \overrightarrow{\mathbf{r}}\right]$ as in the derivation of nonrelativistic oscillator strength sum rules, ${ }^{9}$ the $S_{i}(i=0, \ldots, 4)$ can be calculated exactly. The results are

$$
\begin{aligned}
& S_{0}=\left\langle\psi_{0}\left|r^{2}\right| \psi_{0}\right\rangle, \\
& S_{1}=0, \\
& S_{2}=3 c^{2},
\end{aligned}
$$




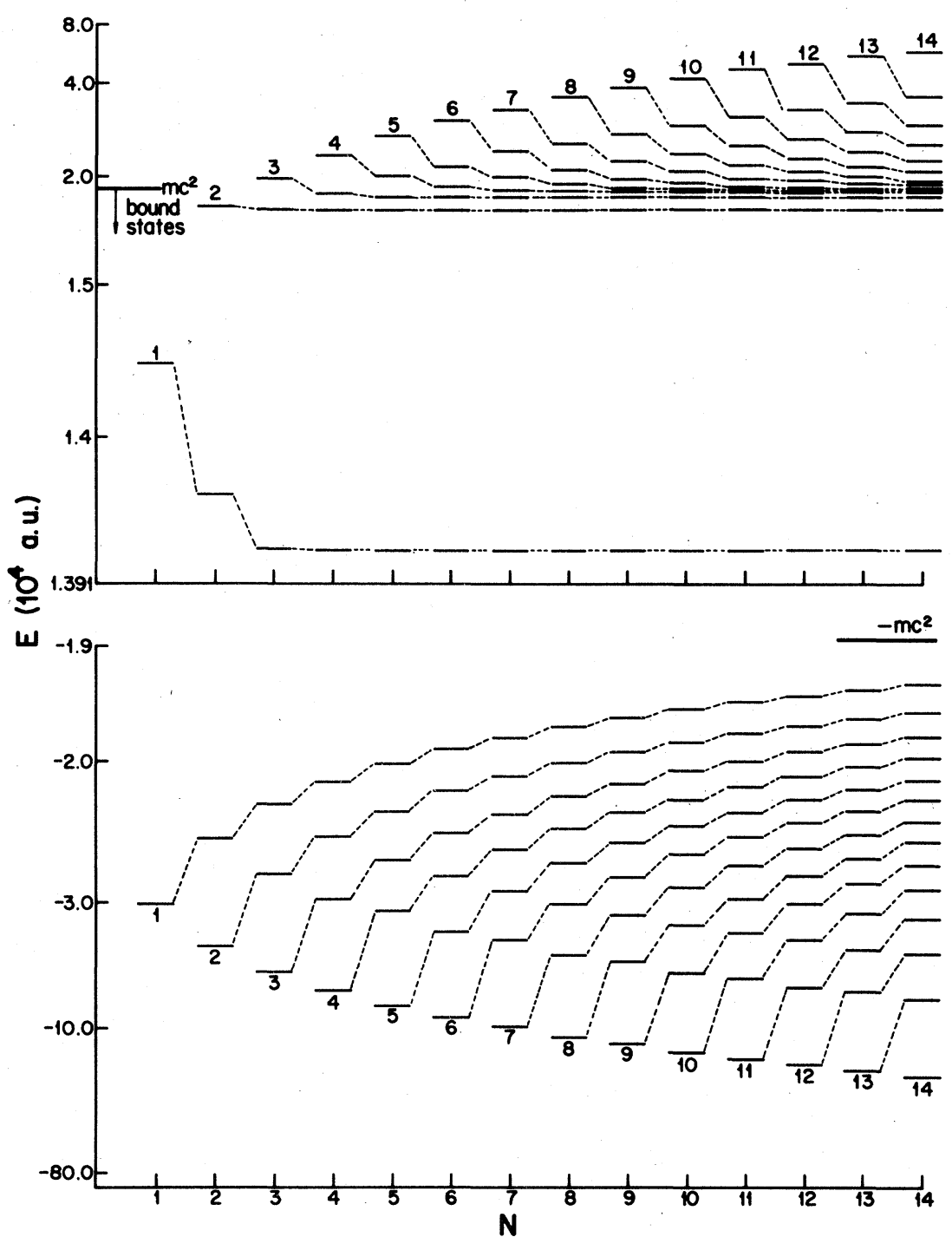

FIG. 1. Distribution of the $n s_{1 / 2}$ variational eigenvalues for $Z=92$ and $\lambda=65.2$ as a function of the size of the basis set. Each basis set of size $2 N$ has $N$-positive eigenvalues in the upper half of the diagram and $N$-negative eigenvalues in the lower half of the diagram. The vertical scale is logarithmic.

$$
\begin{aligned}
& S_{3}=-4 c^{2}\left\langle\psi_{0}|\overrightarrow{\mathrm{r}} \cdot \vec{\nabla} V| \psi_{0}\right\rangle-6 c^{4}\left\langle\psi_{0}|\beta| \psi_{0}\right\rangle, \\
& S_{4}=-8 c^{4}\left\langle\psi_{0}\left|\nabla^{2}\right| \psi_{0}\right\rangle+12 c^{6},
\end{aligned}
$$

and $S_{5}$ diverges for $n s_{1 / 2}$ and $n p_{1 / 2}$ states. The nonrelativistic $S_{4}$ also diverges even though the relativistic $S_{4}$ remains finite. Equation (4.4) for $S_{1}$ shows that the contributions from positive- and negativeenergy states to the nonrelativistic oscillator strength sum (for which $S_{1}=1$ ) exactly cancel. This result was obtained previously by Levinger et al. ${ }^{10}$ Alternatively, the $S_{i}$ can be written in the form

$$
S_{i}=\left\langle\psi_{0}\left|\overrightarrow{\mathbf{r}} \cdot G\left(E_{0}\right)\left(H-E_{0}\right)^{i+1} \overrightarrow{\mathbf{r}}\right| \psi_{0}\right\rangle
$$

with $G\left(E_{0}\right)$ approximated by (4.1). The errors arising from the use of (4.1) with $N=14$ are shown in Table I. All of the errors are small, and extrapolate smoothly to zero as $N$ is increased. This provides strong evidence that the variational representation tends to a complete and accurate description of the Dirac spectrum as the size of the basis set is enlarged.

The sum $S_{-i}$ is related to the dipole polarizability $\alpha_{d} \mathrm{by}^{9}$

$$
\alpha_{d}=\frac{2}{3} S_{-1} \text {. }
$$

Relativistic values of $\alpha_{d} Z^{4}$ (in units of $a_{0}^{4}$ ) are given in Table $I$ for a selection of hydrogenic ions. The values have converged to the number of figures quoted. The exact nonrelativistic value is $\alpha_{d}=4.5 / Z^{4}$. 
TABLE I. Comparison of sum rules [Eqs. (4.3)-(4.7)] with exact values for the $1 s_{1 / 2}$ state, using a $2 \times 14$ term basis set. $\Delta S_{i}=S_{i}$ (exact) $-S_{i}$ (sum rule). For $S_{1}$, the number tabulated is $\Delta S_{1}$ instead of $\Delta S_{1} / S_{1}$. The values of $\gamma$ from (2.9) are 0.99997337 for $Z=1$ and 0.93105942 for $Z=50$.

\begin{tabular}{cccc}
\hline & & \multicolumn{2}{c}{$\Delta S_{i} / S_{i}$} \\
Sum & Exact value & $Z=1$ & $Z=50$ \\
\hline$S_{0}$ & $(\gamma+1)(2 \gamma+1) / 2 Z^{2}$ & $-5 \times 10^{-10}$ & $7 \times 10^{-10}$ \\
$S_{1}$ & 0 & $6 \times 10^{-8}$ & $5 \times 10^{-7}$ \\
$S_{2}$ & $3 / \alpha^{2}$ & $1 \times 10^{-9}$ & $6 \times 10^{-6}$ \\
$S_{3}$ & $-\frac{2}{\alpha^{4}}\left(\gamma+\frac{2}{\gamma}\right)$ & $-3 \times 10^{-11}$ & $2 \times 10^{-5}$ \\
$S_{4}$ & $\frac{4}{\alpha^{6}}\left(\frac{2\left(\gamma^{2}-1\right)(\gamma-2)}{\gamma(2 \gamma-1)}+3\right)$ & $2 \times 10^{-10}$ & $2 \times 10^{-3}$ \\
\hline \hline
\end{tabular}

\section{THE RELATIVISTIC DIPOLE OSCILLATOR STRENGTH SUM}

A long-standing problem of interest in the calculation of the forward photon scattering amplitude at high energies by dispersion theory is the value of the dipole oscillator strength sum ${ }^{10-12}$

$$
S_{1}^{(+)}=\sum_{n+} f_{0, n},
$$

where $n$ runs over only the positive-energy states $n$ (including the continuum). If relativistic and retardation effects are neglected, then the Thomas-Reiche-Kuhn (TRK) sum rule yields the value $S_{1}^{(+)}=1$. The problem of determining the full relativistic value with retardation has been discussed by Payne and Levinger, ${ }^{12}$ and Levinger, Rustgi, and Okamoto. ${ }^{10,11}$ They point out that according to the approximate analytic formulas of Sauter ${ }^{13}$ and Hall, ${ }^{14} S_{1}^{(+)}$diverges logarithmically since the dipole oscillator strength density is approximately proportional to $1 / \omega_{n}$ at very high energies, where $\omega_{n}$ is the transition frequency to the $n$th state. They avoid the divergence by in-

TABLE II. Dipole polarizabilities for hydrogenic ions, obtained with a $2 \times 14$ term basis set. Values not listed can be estimated from the interpolation formula $\alpha_{d} Z^{4}$ $=\frac{9}{2}-\frac{14}{3}(\alpha Z)^{2}+0.53983(\alpha Z)^{4}$.

\begin{tabular}{rl}
$Z$ & $\alpha_{d} Z^{4}\left(a_{0}^{4}\right)$ \\
1 & 4.4997515 \\
5 & 4.4937883 \\
10 & 4.4751644 \\
20 & 4.4008376 \\
30 & 4.2775621 \\
40 & 4.1062474 \\
50 & 3.8881792 \\
60 & 3.6250295 \\
70 & 3.3188659 \\
80 & 2.9721524 \\
90 & 2.5877205 \\
100 & 2.1686483 \\
\hline \hline
\end{tabular}

troducing an arbitrary high-energy cutoff, and find that the summed oscillator strength is insensitive to the cutoff chosen. An advantage of the finite basis set method used here is that it provides a built-in high energy cutoff. Since larger basis sets include progressively higher energy states, the oscillator strength sum is said to converge if the results are independent of the size of the basis set.

Following Grant, ${ }^{15}$ the relativistic dipole oscillator strength from the ground state to an $n p$ state with $J=\frac{1}{2}$ and $\frac{3}{2}$ is given by

$$
f_{0, n}=\frac{1}{3 \alpha^{2}} \sum_{J=1 / 2}^{3 / 2} \frac{(2 J+1)}{\omega_{n J}}\left(\begin{array}{ccc}
\frac{1}{2} & l & J \\
\frac{1}{2} & 0 & -\frac{1}{2}
\end{array}\right)^{2}\left|\bar{M}_{0, n \delta}\right|^{2}
$$

where

$$
\begin{aligned}
& \bar{M}_{\alpha, \beta}= \bar{M}_{\alpha, \beta}^{e}+G \bar{M}_{\alpha, \beta}^{l}, \\
& \bar{M}_{\alpha, \beta}^{e}= \frac{1}{\sqrt{2}}\left[\left(\kappa_{\alpha}-\kappa_{\beta}\right) I_{2}^{+}+2 I_{2}^{-}\right] \\
&-\sqrt{2}\left[\left(\kappa_{\alpha}-\kappa_{\beta}\right) I_{0}^{+}-I_{0}^{-}\right], \\
& \bar{M}_{\alpha, \beta}^{l}= 3 J_{1}+\left(\kappa_{\alpha}-\kappa_{\beta}\right)\left(I_{2}^{+}+I_{0}^{+}\right)-I_{0}^{-}+2 I_{0}^{-}, \\
& I_{L}^{ \pm}(\omega)= \int_{0}^{\infty}\left(g_{\alpha} f_{\beta} \pm f_{\alpha} g_{\beta}\right) j_{L}(\omega r / c) d r, \\
& J_{L}(\omega)= \int_{0}^{\infty}\left(g_{\alpha} g_{\beta}+f_{\alpha} f_{\beta}\right) j_{L}(\omega r / c) d r, \\
& \omega=E(\beta)-E(\alpha),
\end{aligned}
$$

and $j_{L}$ is a spherical Bessel function. For the present calculation, $\alpha=1 s_{1 / 2}$ and $\beta=n p_{J} . \bar{M}_{\alpha, \beta}^{l}$ in (5.3) is the contribution from the longitudinal part of the photon vector potential. This term vanishes identically if exact wave functions are used, making the results independent of the gauge parameter $G$. The choices $G=0$ and $G=\sqrt{2}$ yield the dipole velocity and length forms in the nonrelativistic limit. ${ }^{15}$ The degree to which the present results depend on $G$ provides a check on the accuracy of the calculation.

Numerical values of the relativistic dipole oscillator strength sum with and without retardation are given in Table III for a number of values of $Z$. The values without retardation correspond to $S_{1}^{+}$(no ret) $=\frac{2}{3} \sum_{n+}\left(E_{n}-E_{0}\right)|\langle 0|\overrightarrow{\mathrm{r}}| n\rangle|^{2}$. The sums with $G=\sqrt{2}$ appear to have converged to the number of figures quoted with a $2 \times 14$ term basis set for each of the $n p_{1 / 2}$ and $n p_{3 / 2}$ series of final states. For example, at $Z=82$, the values of $S_{1}^{+}$(ret) are 0.71924 and 0.71932 , respectively, for $2 \times 7$ and $2 \times 14$ term basis sets. To five figures, no further change was found with larger basis sets. A few results with $G=0$ are also given in Table III for comparison. These values are more slowly 
TABLE III. Relativistic dipole oscillator strength sums with and without retardation using a $2 \times 14$ term basis set. $G$ is the gauge parameter appearing in $\mathbf{E q}$. (5.3).

\begin{tabular}{rlll}
\hline & \multicolumn{1}{c}{$S_{1}^{+}$(ret) } & \multicolumn{1}{c}{$S_{1}^{+}$(ret) } & $S_{1}^{+}$(no ret) \\
\hline$Z$ & $G=\sqrt{2}$ & $G=0$ & $G=\sqrt{2}$ \\
1 & 0.9999485 & 0.9999485 & 0.9999556 \\
10 & 0.99490 & 0.99489 & 0.99562 \\
20 & 0.9798 & 0.9797 & 0.9830 \\
30 & 0.9554 & & 0.9633 \\
40 & 0.9236 & & 0.9375 \\
50 & 0.8849 & 0.8845 & 0.9065 \\
60 & 0.8397 & & 0.8710 \\
70 & 0.7882 & & 0.8314 \\
80 & 0.7313 & & 0.7878 \\
82 & 0.7193 & 0.7176 & 0.7784 \\
90 & 0.669 & & 0.7399 \\
100 & 0.60 & & 0.69 \\
\hline \hline
\end{tabular}

convergent with the size of the basis set, but they extrapolate to the same limit as the basis set is increased. To the extent that the figures agree, this demonstrates the gauge invariance of the results.

In common with Payne and Levinger, ${ }^{12}$ the values with retardation lie below those without, but the numerical value of $S_{1}^{+}$(ret) at $Z=82$ is substantially smaller than their result. They obtained 0.86 (Ref. 9) and 0.82 (Ref. 10). Since even our $S_{1}^{+}$(no ret) lies below their values with retardation, it seems likely that their values are too large. However, both calculations suffer from the defect that there may still be a contribution from a very high energy tail which does not show up in the convergence test. Although a sharp high-energy cutoff is not defined, the present calculation includes continuum states up to about 50 times the ionization energy, or about $5 \mathrm{MeV}$ for $Z=82$. It does not seem likely that the present results would get significantly larger with larger basis sets.

\section{DISCUSSION}

We have shown that the Dirac equation with a Coulomb potential can be diagonalized in a suitably chosen finite basis set to obtain a discrete variational representation of the complete Dirac spectrum, including both positive- and negative-energy states. The variational solutions satisfy a variety of sum rules to high accuracy, and can be used to construct an approximate representation of the Dirac Green's function. The oscillator strength sums over positive-energy states calculated in Sec. V are difficult to obtain in any other way. This appears to be a very useful technique for the direct relativistic calculation of atomic properties. Generalizations to systems containing more than one electron are currently under investigation.

\section{ACKNOWLEDGMENT}

Research support from the National Sciences and Engineering Research Council of Canada is gratefully acknowledged. We are grateful to Dr. W. E. Baylis for helpful conversations concerning this work.
${ }^{1}$ E. A. Hylleraas and B. Undheim, Z. Phys. $\underline{65,} 759$ (1930).

${ }^{2}$ Y.-K. Kim, Phys. Rev. 154, 17 (1967); 159, 190E (1967).

${ }^{3}$ J.-P. Desclaux, D. F. Mayers, and F. O'Brien, J. Phys. B4, 631 (1971); J.-P. Desclaux, At. Data Nucl. Data Tables 12, 312 (1973); J.-P. Desclaux, Comput. Phys. Commun. 9, 31 (1975).

${ }^{4}$ S. N. Datta, Chem. Phys. Lett. 74, 568 (1980).

${ }^{5}$ See, for example, G. A. Victor, A. Dalgarno, and A. J. Taylor, J. Phys. B 1, 13 (1968); G. W. F. Drake, G. A. Victor, and A. Dalgarno, Phys. Rev. 180, 25 (1969); G. W. F. Drake, Phys. Rev. Lett. 24, 765 (1970); G. W. F. Drake, Can. J. Phys. 50, 1896 (1972).

${ }^{6}$ For a recent review of the extensive literature on this subject, see W. P. Reinhardt, Comput. Phys. Comm. 17, 1 (1979).

${ }^{7}$ H. Doyle, M. Oppenheimer, and A. Dalgarno, Phys.
Rev. A 11, 909 (1975); P. W. Langhoff, C. T. Corcoran, J. S. Sims, F. Weinhold, and R. M. Glover, Phys. Rev. A 14, 1042 (1976).

${ }^{8}$ A. I. Akhiezer and V. B. Berestetskii, Quantum Electrodynamics, (Wiley, New York, 1965), p. 114.

${ }^{9}$ A. Dalgarno and S. T. Epstein, J. Chem. Phys. 50, 2837 (1969); A. Dalgarno and N. Lynn, Proc. Phys. Soc. London A70, 802 (1957).

${ }^{10} \mathrm{~J}$. S. Levinger, M. L. Rustgi, and K. Okamoto, Phys. Rev. 106, 1191 (1957).

${ }^{11} \mathrm{~J}$. S. Levinger and M. L. Rustgi, Phys. Rev. 103, 439 (1956).

${ }^{12}$ W. B. Payne and J. S. Levinger, Phys. Rev. 101, 1020 (1956).

${ }^{13}$ F. Sauter, Ann. Phys. 11, 454 (1931).

${ }^{14}$ H. Hall, Rev. Mod. Phys. 8 , 358 (1936).

${ }^{15}$ I. P. Grant, J. Phys. B 7 , 1458 (1974). 